\title{
EQUATORS HAVE AT MOST COUNTABLE MANY SINGULARITIES WITH BOUNDED TOTAL ANGLE
}

\author{
Pilar Herreros, Mario Ponce and J. J. P. Veerman
}

Pontificia Universidad Católica de Chile, Facultad de Matemáticas Avda. Vicuña Mackenna 4860, Santiago, Chile; pherrero@mat.puc.cl

Pontificia Universidad Católica de Chile, Facultad de Matemáticas Avda. Vicuña Mackenna 4860, Santiago, Chile; mponcea@mat.puc.cl

Portland State University, Department of Mathematics and Statistics, and CCQCN, Dept. of Physics, University of Crete, 71003 Heraklion, Greece; eerman@pdx.edu

\begin{abstract}
For distinct points $p$ and $q$ in a two-dimensional Riemannian manifold, one defines their mediatrix $L_{p q}$ as the set of equidistant points to $p$ and $q$. It is known that mediatrices have a cell decomposition consisting of a finite number of branch points connected by Lipschitz curves. In the case of a topological sphere, mediatrices are called equators and it can be noticed that there are no branching points, thus an equator is a topological circle with possibly many Lipschitz singularities. This paper establishes that mediatrices have the radial linearizability property. This is a regularity property that implies that at each singular or branching point mediatrices have a geometrically defined derivative in each direction. In the case of equators we show that there are at most countably many singular points and the sum of the angles over all singularities is always finite.
\end{abstract}

\section{Introduction}

Let $M$ be a compact, connected Riemannian manifold. For any distinct points $p, q \in M$ the mediatrix $L_{p q}$ is defined as the set of points with equal distance to $p$ and $q$, that is

$$
L_{p q}=\{x \in M \mid d(x, p)=d(x, q)\},
$$

where $d(\cdot, \cdot)$ is the Riemannian distance in $M$. These geometrical objects can be found in the very beginning of the foundations of geometry. As an illustration, Leibniz proposed to define planes as mediatrices in the space. The first non-trivial approach to mediatrices appears in the book [5] by Busemann, under the name of bisectors. There, the last chapter is devoted mainly to the characterization of metric spaces with flat bisectors. The remarkable result by Busemann is that metric spaces having flat bisectors are (up to a cover) isometric to a finite dimensional Euclidean, hyperbolic, or spherical space. In a more general framework, where one replaces points $p, q$ by disjoint compact sets $P, Q \subset M$, the locus of points with equal distance to $P$ and $Q$, the conflict set, has been extensively studied over the past fifty years, mainly in the case of that $M=\mathbf{R}^{n}$. The work on these sets goes back to the 60's, with the introduction by Milman of the central set (see [8]) and independently by Harry Blum, of the medial axis (see [3]). Since then, an important body of work has been developed in order to understand the structure of these sets in different settings (see for instance $[17,2,12,13,6]$ ). Also, conflict sets and mediatrices have been

https://doi.org/10.5186/aasfm.2017.4251

2010 Mathematics Subject Classification: Primary 53C22; Secondary 53A35.

Key words: Mediatrix, equator, bisectors. 
studied from the topological point of view, and apparently in an independent way, under the name of midsets (see [7]), and equidistant sets (see [16, 11]).

In [1], Bernhard and Veerman show that mediatrices in surfaces have a cell decomposition consisting of a finite number of branch points connected by Lipschitz curves. A branch point in a mediatrix is a point at which (a neighborhood of) the mediatrix is not homeomorphic to an interval. However, singularity points may also appear, where the mediatrix is not a differentiable curve. This paper establishes additional geometric regularity properties of mediatrices in surfaces (see definitions below), that imply that at each branch or singular point mediatrices have a geometrically defined derivative in each direction.

Theorem A. (Short version) Mediatrices in surfaces have the radial linearizability property.

This theorem allows then to define the angle of a singular point as the exterior angle of the vertex it forms.

In the special case where the surface considered is a topological sphere, we will give the special name of equator to mediatrices. We can characterize equators in greater detail. The topology of the sphere restricts the appearance of branch points in equators, as seen in [1], so it is a simple closed curve (a topological circle). In this paper we see that the equator has at most countably many singularities, these are Lipschitz, and the sum of the angles of these singularities has to be finite.

Theorem B. (Short version) Equators are Lipschitz simple closed curves with at most countably many singularities and finite total angle.

The study of mediatrices came about to answer some questions that came up in the study of focal decomposition pioneered by [9] and used in [15]. The simple observation that mediatrices are level sets of the difference of the distance functions to the corresponding points, allows to consider the relationship between the cut locus and mediatrices. Namely, the distance function to a point is differentiable outside the cut locus and hence, the Implicit Function Theorem yields that a mediatrix is differentiable outside the union of the cut locus of the underlying points. It is wellknown (theorems by Singer-Gluck and Itoh) that the cut locus even for a $C^{\infty}$ surface of revolution can be a pretty awful set. In contrast the mediatrix on any $C^{r}, r \geq 3$, surface is locally really surprisingly well-behaved (as we show below).

\section{Preliminaries}

In what follows, $M$ is a two-dimensional compact Riemannian manifold of class $C^{r}, r \geq 3$.

Minimizing geodesics. Let $x \in M$ and $\theta$ in the unit tangent sphere $S_{x} M$. We write $\gamma_{x, \theta}: \mathbf{R} \rightarrow M$ the unit speed geodesic satisfying $\gamma_{x, \theta}(0)=x$ and $\gamma_{x, \theta}^{\prime}(0)=\theta$. For $t^{*}>0$ we say that $\gamma_{x, \theta}$ is a minimizing geodesic between $x$ and $\gamma_{x, \theta}\left(t^{*}\right)$ if the length of $\gamma_{x, \theta}$ equals the infimum of lengths among absolutely continuous curves joining $x$ to $\gamma_{x, \theta}$, that is, length $\left(\left.\gamma_{x, \theta}\right|_{\left[0, t^{*}\right]}\right)=d\left(x, \gamma_{x, \theta}\left(t^{*}\right)\right)$. The triangle inequality yields that $\gamma_{x, \theta}$ is minimizing for every $0<t<t^{*}$.

Polar coordinates. Recall that the exponential map $\exp _{x}$ at $x \in M$ is defined as

$\exp _{x}(v)=\gamma_{x, \hat{v}}(|v|)$, where $v \in T_{x} M, \hat{v}=\frac{v}{|v|}$ if $v \neq 0$, and $\exp _{x}(0)=x$. 
For small $\rho>0$ (less than the injectivity radius $\operatorname{inj} \operatorname{rad}(M)>0$ ), one has that $\left.\exp _{x}\right|_{\{|v|<\rho\}}$ is a diffeomorphism. By fixing a polar coordinates system in $T_{x} M$, one can define (via $\exp _{x}$ ) a local polar coordinates system centered at $x$ on $B_{x, \rho}=$ $\{z \in M \mid d(x, z)<\rho\}$. In that way, a point $z \in B_{x, \rho}, z \neq x$, is identified with a pair $(\theta, d) \in \mathbf{S}^{1} \times(0, \rho)$, such that $z=\gamma_{x, \theta}(d), d=d(x, z)$ (recall that geodesics are locally minimizing curves).

Minimizing directions. For $p \in M, \Theta_{x, p} \subset S_{x} M$ is the set of directions $\theta$ such that $\gamma_{x, \theta}$ is a minimizing curve from $x$ to $p$. We endow $S_{x} M$ with the usual arc-distance in the circle. Since $M$ is complete one has that for $x \neq p$ the set $\Theta_{x, p}$ is non-empty and compact.

Lemma 1. [1] Let $p \neq q \in M$ and $x \in L_{p q}$. Then $\Theta_{x, p} \cap \Theta_{x, q}=\emptyset$.

Proof. Let $\theta \in \Theta_{x, p} \cap \Theta_{x, q}$ and $d=d(x, p)=d(x, q)$. Then $\gamma_{x, \theta}(d)=q=p$, which is impossible.

Lemma 2. Let $x \neq p$, and two convergent sequences $x_{n} \rightarrow x, \theta_{n} \rightarrow \theta$, with $\theta_{n} \in \Theta_{x_{n}, p}$ for every $n$ (we identify $S_{x_{n}} M$ with $S_{x} M$ via a local chart). Then one has $\theta \in \Theta_{x, p}$.

Proof. Let $d_{n}=d\left(x_{n}, p\right)$. Continuity of the distance function implies $d_{n} \rightarrow$ $d(x, p)$. We have $\gamma_{x_{n}, \theta_{n}}\left(d_{n}\right)=p$ for every $n$. Taking the limit we obtain $\gamma_{x, \theta}(d(x, p))=$ $p$, and hence $\theta \in \Theta_{x, p}$.

Corollary 3. If $x \in L_{p q}$, then there exist $\rho_{x}>0$ and $\beta_{x} \in(0, \pi)$ such that

$$
d_{S_{z} M}\left(\Theta_{z, p}, \Theta_{z, q}\right) \geq \beta_{x}
$$

for all $z \in B_{x, \rho_{x}}$.

Proof. Given $x$ we know from Lemma 2 that $\Theta_{x, p} \cap \Theta_{x, q}=\emptyset$. Assume by contradiction that there exist sequences $z_{n} \rightarrow x, \theta_{p, n} \in \Theta_{z_{n}, p}, \theta_{q, n} \in \Theta_{z_{n}, q}$ with $\left|\theta_{p, n}-\theta_{q, n}\right| \rightarrow 0$. Choosing subsequences we can assume that there exists $\theta \in S_{x} M$ such that $\theta_{p, n} \rightarrow \theta, \theta_{q, n} \rightarrow \theta$. Lemma 2 implies that $\theta \in \Theta_{x, p} \cap \Theta_{x, q}$, which is impossible.

For $p \neq q \in M$ and $x \in L_{p q}$, fix a positive orientation in $S_{x} M$. A closed connected set $P$ in $S_{x} M$ is called a pre-wedge at $x$ if its end-points are minimizing directions to $p$ and $q$ respectively, and there are no other minimizing directions to $p$ or $q$ in its interior. Such a set is clearly an arc of the circle, and with a slight abuse of notation we denote it as: $P=\left[\theta_{p}, \theta_{q}\right] \subset S_{x} M$ (or $\left.\left[\theta_{q}, \theta_{p}\right]\right)$. Thus $P$ satisfies:

- $\theta_{p} \in \Theta_{x, p}, \theta_{q} \in \Theta_{x, q}$.

- $\left[\theta_{p}, \theta_{q}\right] \cap\left(\Theta_{x, p} \cup \Theta_{x, q}\right)=\left\{\theta_{p}, \theta_{q}\right\}$.

The midpoint $\hat{\theta}$ of a wedge $P=\left[\theta_{p}, \theta_{q}\right]$ is the unique point contained in the pre-wedge that equidistant from its endpoints.

Lemma 4. [1] There are finitely many pre-wedges at $x \in L_{p q}$.

Proof. Assume $\left(\left[\theta_{p}^{n}, \theta_{q}^{n}\right]\right)_{n \in \mathbf{N}}$ is an infinite sequence of different pre-wedges at $x$. We must have $\lim _{n}\left|\theta_{p}^{n}-\theta_{q}^{n}\right|=0$, since pre-wedges have disjoint interiors. We conclude that the sequences of end-points $\left(\theta_{p}^{n}\right)_{n \in \mathbf{N}},\left(\theta_{q}^{n}\right)_{n \in \mathbf{N}}$ have a common accumulation point $\hat{\theta}$. Compactness of $\Theta_{x, p}$ and $\Theta_{x, q}$ yields that $\hat{\theta}$ belongs to $\Theta_{x, p} \cap \Theta_{x, q}$ which is impossible. 
For $0<\rho<\operatorname{injrad}(M)$ and a pre-wedge $\left[\theta_{p}, \theta_{q}\right] \subset S_{x} M$, we define the corresponding wedge at $x$ of radius $\rho$ as

$$
W_{x, \rho}\left[\theta_{p}, \theta_{q}\right]=\left\{\exp _{x}(r, \theta) \mid 0 \leq r<\rho, \theta \in\left[\theta_{p}, \theta_{q}\right]\right\} \subset M .
$$

In [1] the authors show how the mediatrix $L_{p q}$ is located in $M$ with respect to the wedges at $x \in L_{p q}$ in the following sense:

Theorem 5. (Bernhard and Veerman [1]) Let $M$ be a 2-dimensional compact Riemannian manifold of class $C^{r}, r \geq 3$. For every pair of distinct points $p, q \in M$ the mediatrix $L_{p q}=\{x \in M \mid d(x, p)=d(x, q)\}$ verifies

(1) Let $x \in L_{p q}$. If the cardinalities $\sharp \Theta_{x, p}$ and $\sharp \Theta_{x, q}$ are both 1 , then for small $\rho$ the intersection $L_{p q} \cap B_{x, \rho}$ is a continuous simple curve passing through $x$ and differentiable at $x$.

(2) Let $x \in L_{p q}$ and $0<\rho<\operatorname{injrad}(M)$. The intersection $L_{p q} \cap B_{x, \rho}$ is contained in the finite union of the wedges of radius $\rho$ at $x$.

(3) Let $W_{x, \rho}\left[\theta_{p}, \theta_{q}\right]$ be a wedge at $x \in L_{p q}$. The intersection $L_{p q} \cap W_{x, \rho}\left[\theta_{p}, \theta_{q}\right]$ is a Lipschitz simple curve connecting $x$ to a boundary point of $W_{x, \rho}\left[\theta_{p}, \theta_{q}\right]$. This curve is called a spoke at $x$.

(4) There are finitely many points $x \in L_{p q}$ so that there exists more than two pre-wedges at $x$.

(5) The mediatrix $L_{p q}$ is homeomorphic to a finite and closed simplicial 1-complex.

Notice that (1) above is a direct consequence of the fact that the distance function to a fixed point $p \in M$ is differentiable outside the cut locus of $p$ and a direct application of the Implicit Function Theorem. The Theorem above says that $L_{p q} \cap$ $B_{x, \rho}$ is the finite union of two or more Lipschitz spokes emanating from $x \in L_{p q}$. The goal of this article is to study the behavior of a single spoke as it approaches $x \in L_{p q}$. We show that spokes are radially linearizable in the following sense:

Definition. Let $\phi:[0,1] \rightarrow M$ be a continuous simple curve. We say that $\phi$ is radially linearizable at $x=\phi(0) \in M$ if there exists a direction $\hat{\theta} \in S_{x} M$ such that

$$
\lim _{t \rightarrow 0^{+}} \theta(t)=\hat{\theta}
$$

where $\phi(t)=(\theta(t), d(t))$ in polar coordinates centered at $x$.

Theorem A. (Radial Linearizability of Mediatrices) Let $M$ be a 2-dimensional compact Riemannian manifold of class $C^{r}, r \geq 3$. Let $p \neq q \in M, x \in L_{p q}$, and $W_{x, \rho}\left[\theta_{p}, \theta_{q}\right]$ a wedge at $x$. The spoke $L_{p q} \cap W_{x, \rho}\left[\theta_{p}, \theta_{q}\right]$ is radially linearizable at $x$ in the direction of the midpoint $\hat{\theta}$ of $\left[\theta_{p}, \theta_{q}\right]$.

Notice that this Theorem asserts that $L_{p q} \cap W_{x, \rho}\left[\theta_{p}, \theta_{q}\right]$ is tangent to the bisector of the angle $\angle \theta_{p} \theta_{q}$, and hence, $L_{p q}$ is tangent to the directions of the equidistant set to $\Theta_{x, p}$ and $\Theta_{x, q}$ in $S_{x} M$. A similar result was conjectured in [1] (compare also with $[2])$.

Corollary. Mediatrices in surfaces have no cusp like points.

\section{Proof of Theorem A}

The distance function has one-side directional differential. For $p \in M$, the triangle inequality implies that the function $x \mapsto d(x, p)$ is 1-Lipschitz. Even though $d(x, p)$ is not necessarily differentiable in $M$ (in fact, it is not differentiable 
at the cut locus of $p$ ), for every $x \in M$ this function has a one-sided derivative in every direction. More precisely, let $\theta \in S_{x} M$. The limit

$$
\lim _{t \rightarrow 0^{+}} \frac{d\left(\gamma_{x, \theta}(t), p\right)-d\left(\gamma_{x, \theta}(0), p\right)}{t}
$$

does exist. This fact, and the explicit formula for the limit, is a well known fact that can be deduced from the first variation formula. The following Proposition often appears as a folkloric result, and a proof can be found in the Plaut's work [10, p. 844]:

Proposition 6. Using the above notation, one has

$$
\lim _{t \rightarrow 0^{+}} \frac{d\left(\gamma_{x, \theta}(t), p\right)-d\left(\gamma_{x, \theta}(0), p\right)}{t}=-\cos \left(d_{S_{x} M}\left(\theta, \Theta_{x, p}\right)\right),
$$

where $d_{S_{x} M}(\cdot, \cdot)$ is the Riemannian distance on the unit circle between $\theta$ and the compact set of minimizing directions from $x$ to $p$.

Lemma 7. Let $g:[0,1) \rightarrow \mathbf{R}$ be a continuous function and $c \in \mathbf{R}$. Assume that the one-sided derivative exists and verifies

$$
g_{+}^{\prime}(t) \equiv \lim _{s \rightarrow 0^{+}} \frac{g(t+s)-g(t)}{s}>c
$$

for every $t \in[0,1)$. Then $g(t)>g(0)+c t$ for every $t \in(0,1)$.

Proof. By considering the function $g(t)-c t$ we can reduce the problem to the case $c=0$. For every $t \in[0,1)$ there exists $\delta_{t}>0$ such that $g(t+s)-g(t)>0$ for $0<s<\delta_{t}$. Hence $g$ can not have local maximal points. As $g$ is continuous, the only possibility is that $g$ is strictly increasing.

In what follows we use the notation of the statement of the Main Theorem, that is, we fix a pre-wedge $\left[\theta_{p}, \theta_{q}\right]$ at $x \in L_{p q}$, write $\hat{\theta}$ the midpoint of $\left[\theta_{p}, \theta_{q}\right]$, and $\rho_{x}>0$ given by Corollary 3 .

Lemma 8. If $L_{p q} \cap W_{x, \rho}\left[\theta_{p}, \theta_{q}\right] \cap \gamma_{x, \theta}\left(\left[0, \rho_{x}\right)\right)$ contains a sequence of points converging to $x$ then $\theta=\hat{\theta}$.

Proof. Define $f_{p q}(z)=d(z, q)-d(z, p)$. Hence $L_{p q}=f_{p q}^{-1}(0)$. Proposition 6 allows to deduce that $f_{p q}$ has one-side differential at $x$ in the direction of $\theta$ equal to $-\cos \left|\theta-\tilde{\theta}_{p}\right|+\cos \left|\theta-\tilde{\theta}_{q}\right|$ for some $\tilde{\theta}_{p} \in \Theta_{x, p}, \tilde{\theta}_{q} \in \Theta_{x, q}$. If the restriction of $f_{p q}$ to the ray $\gamma_{x, \theta}$ has roots accumulating at $x$ then this one-side differential must be equal to zero. The only possible case is $\tilde{\theta}_{p}=\theta_{p}, \tilde{\theta}_{q}=\theta_{q}$ (otherwise minimizing geodesics will cross) and it follows that the only solution is $\theta=\hat{\theta}$.

This Lemma says that whenever a spoke crosses infinitely many times a geodesic ray emanating from $x$, and the crossing points approach $x$, then the geodesic ray necessarily points in the direction of the bisector of the corresponding pre-wedge. The next result estimates for how much the points in the geodesic ray $\gamma_{x, \hat{\theta}}$ fail to belong to the mediatrix $L_{p q}$.

Lemma 9. Given $p$ and $q$, then for each $x \in L_{p q}$ there exists a positive function $\eta$ such that

$$
\left|d\left(\gamma_{x, \hat{\theta}}(t), q\right)-d\left(\gamma_{x, \hat{\theta}}(t), p\right)\right| \leq \eta(t) t \quad \text { for } 0 \leq t<\rho,
$$

and $\lim _{t \rightarrow 0} \eta(t)=0$. 
Proof. The function $t \mapsto f_{p q}\left(\gamma_{x, \hat{\theta}}(t)\right)$ together with its first (right directional) derivative vanish at $t=0$ and the result follows.

The next result says that for points that fail to belong to $L_{p q}$ by few, we can find a genuine point in the mediatrix close to it. This result is inspired by Section 4.2 of [11].

Lemma 10. Let $x \in L_{p q}$ and $\rho_{x}>0, \beta_{x} \in(0, \pi)$ given by Corollary 3. There exists $\varepsilon_{x}>0$ such that for all $z \in B_{x, \rho_{x} / 2}$ that verifies $|d(z, q)-d(z, p)|<\varepsilon_{x}$ there exists $z^{*} \in L_{p q}$, and

$$
d\left(z, z^{*}\right)<\frac{|d(z, q)-d(z, p)|}{1-\cos \left(\beta_{x}\right)}
$$

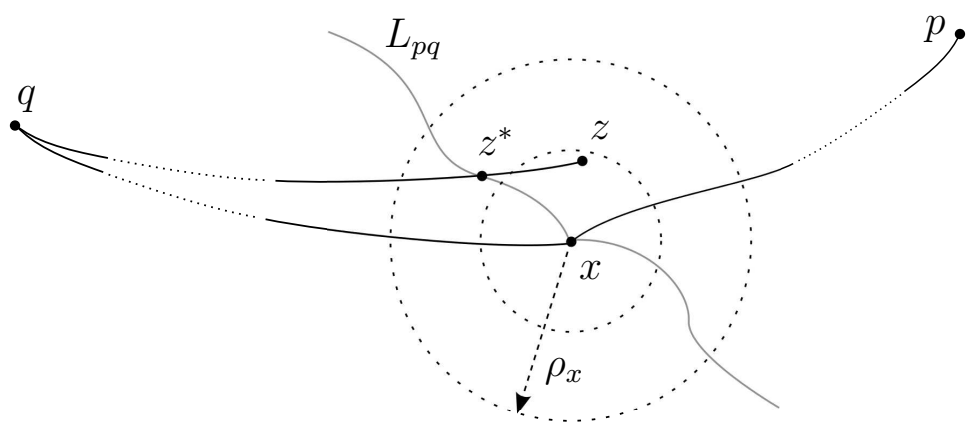

Figure 1 .

Proof. Let's assume that $d(z, q)>d(z, p)$. Pick $\theta \in \Theta_{z, q}$ (this also gives $\left.\theta \notin \Theta_{z, p}\right)$. By moving continuously along the geodesic ray $z(t) \equiv \gamma_{z, \theta}(t)$ we must encounter a point $z^{*} \equiv z\left(t^{*}\right)$ in the mediatrix $L_{p q}$ (since $\gamma_{z, \theta}$ reaches $q$ ). We estimate the distance $t^{*}$ between $z$ and $z^{*}$. Note that since $d(x, z)<\rho_{x} / 2$ then by construction $d(x, z(t))<\rho_{x}$ at least for $t \leq \rho_{x} / 2$.

By Proposition 6 we have

$$
\lim _{s \rightarrow 0^{+}} \frac{d(z(t+s), p)-d(z(t), p)}{s}=-\cos \left(d_{S_{z(t)} M}\left(z^{\prime}(t), \Theta_{z(t), p}\right)\right) .
$$

For every $t \in\left[0, \rho_{x} / 2\right]$ one has $z^{\prime}(t) \in \Theta_{z(t), q}$ and so Corollary 3 implies that there is a $\beta_{x} \in(0, \pi)$ so that

$$
\cos \left(d_{S_{z(t)} M}\left(z^{\prime}(t), \Theta_{z(t), p}\right)\right) \leq \cos \beta_{x}
$$

Now Lemma 7 implies that for every $t \in\left(0, \rho_{x} / 2\right]$

$$
d(z(t), p) \geq d(z, p)-t \cos \beta_{x} .
$$

We also have that

$$
d(z(t), q)=d(z, q)-t
$$

Putting these two together we have that

$$
\begin{aligned}
& d(z(0), q)-d(z(0), p)>0, \\
& d(z(t), q)-d(z(t), p) \leq d(z(0), q)-d(z(0), p)-t\left(1-\cos \left(\beta_{x}\right)\right) .
\end{aligned}
$$

Now, if we choose $\varepsilon_{x} \equiv \frac{1}{2} \rho_{x}\left(1-\cos \left(\beta_{x}\right)\right)>0$, then $t_{0}=\frac{d(z, q)-d(z, p)}{1-\cos \left(\beta_{x}\right)}$ is in $\left(0, \rho_{x} / 2\right]$ so that the last inequality holds at $t_{0}$ giving $d(z(t), q)-d(z(t), p) \leq 0$. Therefore, by continuity of the distance function, we have $t^{*} \leq t_{0}$ and the Lemma is verified.

Proof of Theorem A. Pick $x \in L_{p q}$ and a wedge $W_{x, \rho}\left[\theta_{p}, \theta_{q}\right]$. Reasoning by contradiction let's suppose that there exists $\tau>0$ such that the spoke contains a 
sequence of points $x_{n}$ converging to $x$ outside the cone $[\hat{\theta}-\tau, \hat{\theta}+\tau] \times(0, \rho)$ (in polar coordinates centered at $x$ ). Lemmas 9 and 10 assert that the spoke contains a sequence of points inside the cone as it approaches $x$, since the spoke passes through a point at a distance less than $\left(1-\cos \beta_{x}\right)^{-1} \eta(t) t$ from $\gamma_{x, \hat{\theta}}(t)$ and thus inside the cone for $t$ sufficiently small. By the continuity of the spoke, it must cross $\gamma_{x, \hat{\theta}+\tau}$ (or $\left.\gamma_{x, \hat{\theta}-\tau}\right)$ an infinite number of times, and Lemma 8 says that this is impossible unless $\tau=0$.

\section{Equators and the Pugh-Tangerman example}

Let $M$ be a simply connected 2-dimensional Riemannian manifold of class $C^{r}, r \geq$ 3 (a sphere). For $p \neq q \in M$ we already know that there are at most finitely many points $x \in L_{p q}$ such that $L_{p q}$ is not locally homeomorphic to an interval at $x$ (that is, there are more than two wedges at $x$ ). Let us call such a point a branching point (and a simple point to a non-branching point). For a simple point $x$ the Theorem A asserts that $L_{p q}$ has two well defined directions $\hat{\theta}_{1}, \hat{\theta}_{2} \in S_{x} M$ at $x$ (the bisectors of the two pre-wedges at $x$, or in other words, the equidistant set in $S_{x} M$ of the sets of minimizing directions from $x$ to $p$ and $q$ respectively). We define the angle at $x$ as

$$
\angle_{L_{p q}}(x)=|\pi-| \hat{\theta}_{1}-\hat{\theta}_{2}|| \text {. }
$$

If $L_{L_{p q}}(x) \neq 0$ we say that $x$ is a Lipschitz singularity of the equator $L_{p q}$.

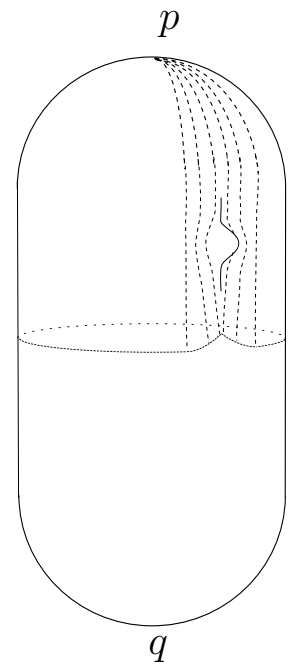

Figure 2. The Pugh-Tangerman example.

Pugh-Tangerman example. A very illustrative example is what has come to be known as Pugh's Cigar $C$ embedded in $\mathbf{R}^{3}$. It consists of the cylinder given by $x^{2}+y^{2}=1$ plus two (unit) hemispheres glued to each of the boundaries. Let $p$ be its North Pole and $q$ its South Pole. The equator is given by the circle at $z=0$.

We can obtain an arbitrarily smooth cigar by smoothing around $|z|= \pm 1$. If we add a "bump" in the flat "northern" part of the cigar, keeping $C$ as a smooth manifold, is easy to see that the equator will develop a Lipschitz singularity near the closest point to the bump. Adding a sequence of bumps in the northern part that do not intersect each other we can create a cigar whose equator will have countably many Lipschitz singularities. 
In what follows we want to show that this is, in some sense, the worst possible behavior for equators. This proposition is inspired on conversations in the 1990's by Pugh and Tangerman with Veerman at Rockefeller University.

Theorem B. Let $M$ be a sphere of class $C^{r}, r \geq 3$ and $p \neq q \in M$. Then the equator $L_{p q}$ verifies

(1) $L_{p q}$ is a Lipschitz simple closed curve.

(2) There are at most countably many Lipschitz singularities in $L_{p q}$. Moreover the angles at these singularities form an absolutely summable sequence, that is

$$
\sum_{x \in L_{p q}} L_{L_{p q}}(x)<\infty
$$

Proof. Simple closed follows from earlier results in [1]. The novelty here is the Lipchitz property. The fact that the equator is a simple closed curve implies that at each $x \in L_{p q}$ there are exactly two spokes, and thus exactly two wedges. By Theorem A, the mediatrix is tangent to the bi-sectors of these two wedges, and thus the singularity is Lipschitz.

We saw that at a given point $x \in L_{p q}$ there are exactly two wedges each of which is bounded by two minimizing geodesics $\gamma_{x, p}$ and $\gamma_{x, q}$. Take the complement of the closure of these wedges. If it is the empty set, the equator is differentiable at $x$. If not, then (a) that complement is a maximal wedge shaped set bounded by two minimizing geodesics from $x$ to $p$, or (b) bounded two minimizing geodesics from $x$ to $q$, or (c) both of these occur. In case (a) let us denote by $\mathcal{J}_{x, p}$ the open domain bounded by the indicated geodesic rays from $x$ to $p$ and by $\mu_{x, p}$ the opening angle at $x$ of that wedge-shaped figure. Similarly we have $\mathcal{J}_{x, q}$ and $\mu_{x, q}$ for case (b). Case (c) means that both $\mathcal{J}_{x, p}$ and $\mathcal{J}_{x, p}$ are non-empty. Notice that $\mathcal{J}_{x, p}=\emptyset$ if and only if $\sharp \Theta_{x, p}=1$.

If we have two minimizing geodesics from $x_{1}, x_{2} \in L_{p q}$ to $p$ (or $q$ ) that intersect at a point $z$, we would have two different geodesics $\gamma_{1}$ and $\gamma_{2}$ from $z$ to $p$ forming a non-zero angle. Since both $\gamma_{1}$ and $\gamma_{2}$ are minimizing they have the same length $d(z, p)$, but by triangle inequality

$$
d\left(x_{1}, z\right)+\text { length }\left(\gamma_{2}\right)>d\left(x_{1}, p\right)=d\left(x_{1}, z\right)+\text { length }\left(\gamma_{1}\right),
$$

thus these geodesics do not intersect. Therefore the collection (as $x$ runs on $L_{p q}$ ) of Jordan domains $\mathcal{J}_{x, p}, \mathcal{J}_{x, q}$ are pairwise disjoint. We conclude that there are at most countably many points $x \in L_{p q}$ such that $\sharp \Theta_{x, p}$ or $\sharp \Theta_{x, q}$ are different from 1 . A direct examination yields that

$$
2 L_{L_{p q}}(x)=\left|\mu_{x, p}-\mu_{x, q}\right| .
$$

The Gauss-Bonnet Theorem applied to $\mathcal{J}_{x, p}$ gives

$$
\int_{\mathcal{J}_{x, p}} k(w) d w=\mu_{x, p}+\alpha_{x, p}
$$

where $\alpha_{x, p}$ is the angle of the landing geodesic rays make at $p$ (note that $\mu_{x, p}$ is the angle they make at $x)$, and $k(w)$ is the curvature at $w \in M$. We estimate

$$
\begin{aligned}
\sum_{x \in L_{p q}} L_{L_{p q}}(x) & <\frac{1}{2} \sum_{x \in L_{p q}}\left(\int_{\mathcal{J}_{x, p}}|k(w)| d w+\int_{\mathcal{J}_{x, q}}|k(w)| d w+\left|\alpha_{x, p}\right|+\left|\alpha_{x, q}\right|\right) \\
& \leq \frac{1}{2}\left(\int_{M}|k(w)| d w+4 \pi\right) .
\end{aligned}
$$


This also shows that $\angle_{L_{p q}}(x)$ is bounded, giving a Lipschitz constant for all singularities, and therefore the whole curve.

Acknowledgments. Pilar Herreros acknowledges the financial support of the research project FONDECYT 1170665. Mario Ponce and J. J. P. Veerman acknowledge the financial support of the research project FONDECYT 1140988. J. J. P. Veerman's research was also partially supported by the European Union's Seventh Framework Program (FP7-REGPOT-2012-2013-1) under grant agreement n316165.

\section{References}

[1] Bernhard, J., and J. J. P. Veerman: The topology of surface mediatrices. - Topology Appl. $154: 1,2007,54-68$.

[2] Birbrair, L., and D. Siersma: Metric properties of conflict sets. - Houston J. Math. 35:1, 2009, 73-80.

[3] Blum, H.: A transformation for extracting new descriptors of shape. - In: Models for the perception of speech and visual form, W. Wathen-Dunn, ed., MIT Press, Cambridge, MA, $1967,362-380$.

[4] Burago, D., Y. Burago, and S. Ivanov: A course in metric geometry. - Grad. Stud. Math. 33, Amer. Math. Soc., Providence, RI, 2001.

[5] Busemann, H.: The geometry of geodesics. - Academic Press, Inc., New York, 1955.

[6] Damon, J.: Smoothness and geometry of boundaries associated to skeletal structures. I. Sufficient conditions for smoothness. - Ann. Inst. Fourier (Grenoble) 53:6, 2003, 1941-1985.

[7] Loveland, L. D.: When midsets are manifolds. - Proc. Amer. Math. Soc. 61:2, 1976, 353-360.

[8] Milman, D.: The central function of the boundary of a domain and its differentiable properties. - J. Geom. 14:2, 1980, 182-202.

[9] Peixoto, M. M., and R. Thом: Le point de vue énumératif dans les problèmes aux limites pour les équations différentielles ordinaires. I: Quelques exemples. - C. R. Math. Acad. Sci. Paris I 303, 1986, 629-632. Also: C. R. Math. Acad. Sci. Paris I 307, 1988, 197-198.

[10] Plaut, C.: Metric spaces of curvature $\geq k$. - In: The handbook of geometric topology, NorthHolland, Amsterdam, 2002, 819-898.

[11] Ponce, M., and P. Santibáñez: On equidistant sets and generalized conics: the old and the new. - Amer. Math. Monthly 121:1, 2014, 18-32.

[12] Sotomayor, J., D. Siersma, and R. Garcia: Curvatures of conflict surfaces in Euclidean 3-space. - In: Geometry and topology of caustics - CAUSTICS '98 (Warsaw), Banach Center Publ. 50, 1999, 277-285.

[13] Van Manen, M.: Conflict sets, ortonomics, pedals and billiards as canonical relations. - Math. Proc. Cambridge Philos. Soc. 141, 2006, 339-353.

[14] Veerman, J. J. P., and J. Bernhard: Minimally separating sets, mediatrices, and Brillouin spaces. - Topology Appl. 153:9, 2006, 1421-1433.

[15] Veerman, J. J. P., M. M. Peixoto, A. C. Rocha, and S. Sutherland: Brillouin zones. Comm. Math. Phys. 212:3, 2000, 725-744.

[16] Wilker, J. B.: Equidistant sets and their connectivity properties. - Proc. Amer. Math. Soc. $47,1975,446-452$.

[17] Yomdin, Y.: On the local structure of a generic central set. - Compositio Math. 43:2, 1981, $225-238$. 